\section{Fast-track revision hip arthroplasty: a multicenter cohort study on 1,345 elective aseptic major component revision hip arthroplasties}

\author{
Martin LINDBERG-LARSEN ${ }^{1-3}$, Pelle Baggesgaard PETERSEN ${ }^{4}$, Yasemin CORAP ${ }^{1,2}$, \\ Kirill GROMOV ${ }^{3,5}$, Christoffer Calov JøRGENSEN ${ }^{4}$ and Henrik KEHLET ${ }^{3,4}$ on behalf of the \\ Centre for Fast-track Hip and Knee Replacement Collaborating Group ${ }^{\text {a }}$
}

\author{
${ }^{1}$ Orthopaedic Research Unit, Department of Orthopaedic Surgery and Traumatology, Odense University Hospital; \\ ${ }^{2}$ Department of Clinical Research, University of Southern Denmark; ${ }^{3}$ Centre for Fast-track Hip and Knee Arthroplasty, \\ Copenhagen, Denmark; ${ }^{4}$ Section for Surgical Pathophysiology, Rigshospitalet, Copenhagen, Denmark; ${ }^{5}$ Department of \\ Orthopedics, Hvidovre Hospital, Hvidovre, Denmark \\ a The patients in this study underwent revision knee surgery at the centers participating in the Centre for Fast-track Hip and \\ Knee Replacement Collaboration. The surgeons in the collaborating group listed below reviewed and approved the final manu- \\ script. Members of the Centre for Fast-track Hip and Knee Replacement Collaborative Group: Frank MADSEN, Department \\ of Orthopedics, Aarhus University Hospital, Aarhus, Denmark; Torben Bæk HANSEN, Department of Orthopedics, Regional \\ Hospital Holstebro and University of Aarhus, Holstebro, Denmark; Claus VARNUM, Department of Orthopedic Surgery, \\ Lillebaelt Hospital - Vejle, University Hospital of Southern Denmark, Denmark; Søren SOLGAARD, Department of Orthopedics, \\ Gentofte University Hospital, Copenhagen, Denmark; Torben BECK, Department of Orthopaedic Surgery, Copenhagen \\ University Hospital Bispebjerg, Copenhagen NV, Denmark. \\ Correspondence: martin.lindberg-larsen@rsyd.dk \\ Submitted 2021-12-03. Accepted 2022-02-02.
}

Background and purpose - Data on application of fasttrack/enhanced recovery protocols in revision hip arthroplasty (R-THA) surgery is scarce. We report length of stay (LOS), risk of LOS $>5$ days, and readmission $\leq 90$ days after revision hip arthroplasty in centers with a well-established fast-track protocol in both primary and revision procedures.

Patients and methods - This is an observational cohort study from the Centre for Fast-track Hip and Knee Replacement and the Danish Hip Arthroplasty Register. Consecutive elective aseptic major component revision hip arthroplasties from 6 dedicated fast-track centers from 2010 to 2018 were included.

Results - 1,345 R-THAs were analyzed, including $23 \%$ total revisions, $52 \%$ acetabular component revisions, and $25 \%$ femoral component revisions. Mean age was 70 years (SD 12) and $61 \%$ were female. Median LOS was 3 days (interquartile range [IQR] 2-6), decreasing from median 6 (IQR 3-10) days in 2010 to 2 (IQR 1-4) days in 2018. The 90 -day readmission rate was $20 \%$, but showed a fluctuating and increasing trend from 13\% in 2010 to $28 \%$ in 2018. Risk factors for LOS $>5$ days and readmission were use of walking aid, preoperative hemoglobin $\leq 13 \mathrm{~g} / \mathrm{dL}$, pharmacological treated psychiatric disorder, age $\geq 80$ years, age 70-79 years (only LOS $>5$ days), cardiac disease (only LOS $>5$ days), pulmonary disease (only readmission), BMI $\geq 35$ (only LOS $>5$ days) and $\geq 1$ previous revision (only LOS $>$ 5 days).
Interpretation - LOS decreased to median 2 days at the end of the study period, but the 90 days readmission risk remained high (>20\%). Several risk factors for postoperative complications were identified, suggesting that at-risk patients should be treated using an extended fast-track/ enhanced recovery protocol focusing on preoperative optimization and postoperative monitoring as well as surgical techniques to reduce hip dislocations.

To our knowledge, only 1 previous study has investigated the application of a fast-track/enhanced recovery protocol in revision hip arthroplasty procedures in the same way as has been used in primary total hip arthroplasty (THA). Joseph et al. (1) reported data on 126 revision THA (R-THA) procedures (including 21 septic revisions) from a single center performed by a single surgeon with a length of hospital stay of 5 days for non-septic procedures.

Nationwide data on aseptic R-THA procedures from Denmark (2009-2011) showed that fast-track protocols had already been implemented with median LOS of 5 days but a readmission rate of $18 \%$ (2). Hence, data on patient safety and the role of patient characteristics in fast-track revision hip arthroplasty is limited.

We report LOS, risk of LOS $>5$ days and readmission $\leq 90$ days after elective aseptic major component R-THA in centers 
with a well-established fast-track protocol in both primary and revision procedures.

\section{Patients and methods}

\section{Study design}

This is an observational cohort study on patients from the Centre for Fast-track Hip and Knee Replacement (www.fthk. $\mathrm{dk}$ ). The STROBE guideline for reporting of an observational study was followed. The revision procedures reported on in this study were also part of the study cohort of another study specifically analyzing the risk of venous thromboembolism after both revision hip and knee arthroplasty procedures (3).

\section{Setting}

We included a consecutive cohort of unselected and elective fast-track aseptic major component R-THAs from 6 dedicated fast-track centers from January 11, 2010, to June 29, 2018. The dedicated fast-track centers agreed to use similar fast-track protocols in elective revision procedures comparable to the perioperative care process for primary THA (4) and where the median LOS in 2018 was 1 day (5). All centers were high-volume centers in both primary and revision procedures according to Danish standards. The number of revision procedures in the centers ranged from 123 to 384 .

The fast-track protocol includes planned use of multimodal opioid-sparing analgesia, intended early mobilization $(<6$ hours postoperatively), and discharge to own home based on functional discharge criteria (independent in personal care, able to walk with crutches, able to get in and out of bed and into and up from a chair, and sufficient oral pain treatment) (6). 1 gram of tranexamic acid was administered intravenously during surgery in all centers and a repeated postoperative dose was used in 4 centers. Intraoperative high-volume local infiltration analgesia (LIA) was not used. Postoperative thromboprophylaxis was administered 6 to 8 hours after surgery and only used during primary admission if LOS $\leq 5$ days in all centers during the whole study period. If $\operatorname{LOS}>5$ days national recommendations (7) were followed and thromboprophylaxis was used up to 14 days in the period from 2010 to 2016 and for 6 to 10 days from 2016 to 2018 (3). There was no mutual guideline on type of anesthesia, use of preoperative high-dose glucocorticoid (only used routinely in 4 of 6 centers and not for the whole study period), and use of surgical drain as this was based on surgeon and center preferences.

All procedures were performed with a posterior approach.

\section{Data sources}

All elective aseptic major component revision hip arthroplasty procedures were identified from the Danish Hip Arthroplasty Register (DHR) (7). Furthermore, information on type of revision, indications, previous revisions, and duration of surgery was obtained from DHR.

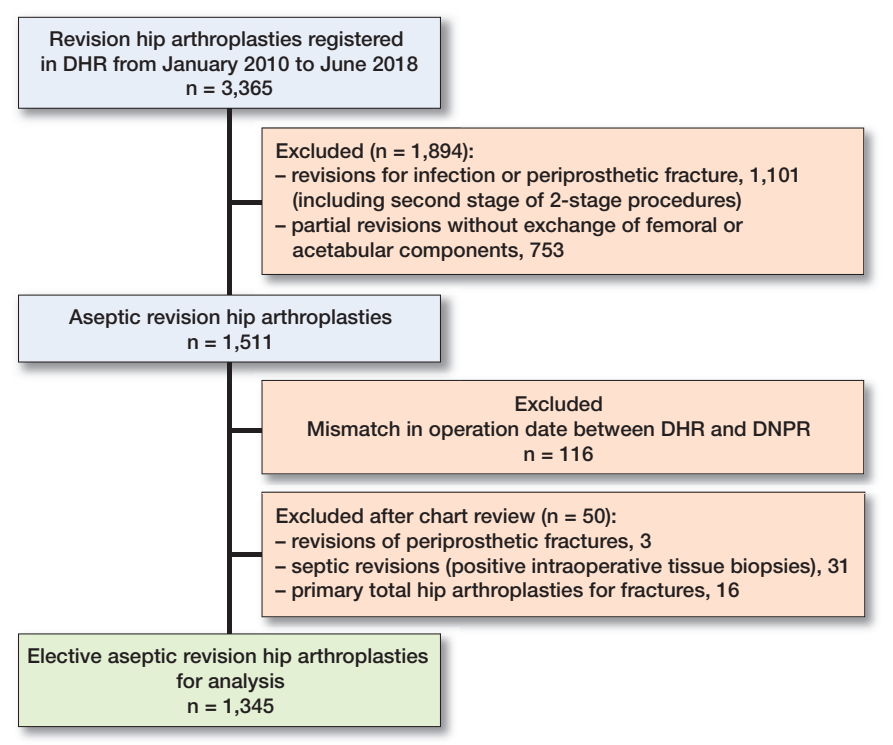

Figure 1. Study cohort: flowchart showing inclusions and exclusions.

Data on preoperative comorbidity and patient characteristics was prospectively collected from patients within 1 month before surgery using self-completed questionnaires with staff available for assistance. Validation of the consistency of the preoperative patient questionnaire has been performed previously using matched patient medical records (8).

Supplementary data on pharmacologically treated diabetes and psychiatric disorders was obtained from the Danish National Database of Reimbursed Prescriptions (9).

Data on LOS, readmissions, and mortality within 90 days postoperatively was obtained from the Danish National Patient Registry (DNPR), with > 99\% completeness of follow-up (10). Data on specific complications was based on review of discharge summaries or patient records in the case of LOS > 5 days and 90-day readmission or mortality. Review of the records was done by YC, PBP, and MLL.

\section{Outcomes}

The primary objective was to investigate LOS, and risk of complications within 90 days postoperatively by analyzing causes of prolonged LOS ( $>5$ days) and readmissions. A LOS $>5$ days was considered prolonged based on the median LOS of 5 days found after aseptic revision hip arthroplasties nationwide in Denmark (2). We analyzed LOS (number of postoperative overnight stays, including transferals to other departments and hospitals) and readmissions within 90 days postoperatively (requiring 1 overnight stay and being potentially related to index procedure) as well as 90-day incidence of complications not requiring overnight stay.

\section{Patients and surgical procedures}

Data on 3,118 R-THA performed in the 6 centers from January 2010 to June 2018 was acquired. We excluded all non- 
Table 1. Patient and surgical characteristics. Data given as count (\%) or mean (SD)

\begin{tabular}{|c|c|c|c|c|}
\hline Factor & $\begin{array}{l}\text { All } \\
\text { revisions }\end{array}$ & $\begin{array}{l}\text { Total } \\
\text { revisions }\end{array}$ & $\begin{array}{l}\text { Acetabular } \\
\text { component } \\
\text { revisions }\end{array}$ & $\begin{array}{l}\text { Femoral } \\
\text { component } \\
\text { revisions }\end{array}$ \\
\hline No. of cases & 1,345 & $313(23)$ & $699(52)$ & $333(25)$ \\
\hline \multicolumn{5}{|l|}{ Patient characteristics } \\
\hline Mean age, years (SD) & $70(12)$ & $69(13)$ & $70(12)$ & $71(12)$ \\
\hline Female sex & $816(61)$ & $178(57)$ & $456(65)$ & $182(55)$ \\
\hline $\begin{array}{l}\text { BMI } \geq 35 \\
\quad \text { Missing } 59 \text { (4) }\end{array}$ & $86(6)$ & $21(7)$ & $42(6)$ & $23(7)$ \\
\hline $\begin{array}{l}\text { Use of walking aid } \\
\text { Missing } 105 \text { (8) }\end{array}$ & $546(41)$ & $139(44)$ & 260 (37) & $147(44)$ \\
\hline \multicolumn{5}{|l|}{ Diabetes } \\
\hline Insulin dependent & $19(1)$ & $4(1)$ & $7(1)$ & $8(2)$ \\
\hline Non-insulin dependent & $80(6)$ & $12(4)$ & $45(6)$ & $23(7)$ \\
\hline $\begin{array}{l}\text { Cardiac disease } \\
\text { Missing } 30(2)\end{array}$ & $253(19)$ & $68(22)$ & $127(18)$ & $58(17)$ \\
\hline $\begin{array}{l}\text { Pulmonary disease } \\
\text { Missing } 22(2)\end{array}$ & $151(11)$ & $43(14)$ & $70(10)$ & $38(11)$ \\
\hline $\begin{array}{l}\text { Pharmacologically treated } \\
\text { psychiatric disorder }\end{array}$ & $216(16)$ & $46(15)$ & $113(16)$ & $57(17)$ \\
\hline $\begin{array}{l}\text { Preoperative } \mathrm{Hb} \leq 13 \mathrm{~g} / \mathrm{dL} \\
\text { Missing } 191(14)\end{array}$ & $396(29)$ & $85(27)$ & $200(29)$ & $111(33)$ \\
\hline \multicolumn{5}{|l|}{ Surgical characteristics } \\
\hline $\begin{array}{l}\text { Mean duration of surgery, } \\
\text { minutes (SD) }\end{array}$ & $118(58)$ & $160(69)$ & $93(37)$ & $134(56)$ \\
\hline $\begin{array}{l}\text { Previously revised } \\
\text { Missing } 25(2)\end{array}$ & $269(20)$ & $47(15)$ & $143(21)$ & $79(24)$ \\
\hline \multicolumn{5}{|l|}{ Femoral head size, $\mathrm{mm}$} \\
\hline$\leq 28^{a}$ & $181(14)$ & $20(6)$ & $125(18)$ & $36(11)$ \\
\hline 32 & $315(23)$ & $45(14)$ & $157(23)$ & $113(34)$ \\
\hline 36 & $817(61)$ & $238(76)$ & $398(57)$ & $181(54)$ \\
\hline $\begin{array}{l}>36 \\
\text { Missing } 13(1)\end{array}$ & $19(19)$ & $7(2)$ & $9(1)$ & $37(1)$ \\
\hline \multicolumn{5}{|l|}{ Type of implant liner } \\
\hline Non-constrained liner 1 & $1,159(86)$ & 291 (93) & $574(82)$ & $294(88)$ \\
\hline Constrained liner & $43(3)$ & $4(1)$ & $30(4)$ & $9(3)$ \\
\hline No liner & $14(1)$ & 0 & $4(1)$ & $10(3)$ \\
\hline $\begin{array}{l}\text { Dual mobility } \\
\text { Missing } 13(1)\end{array}$ & $116(9)$ & $14(5)$ & $89(13)$ & $13(4)$ \\
\hline \multicolumn{5}{|l|}{ Type of anesthesia } \\
\hline Spinal/epidural & $874(65)$ & $193(62)$ & $468(67)$ & $213(64)$ \\
\hline General anesthesia & 435 (32) & 109 (35) & $218(31)$ & $108(32)$ \\
\hline $\begin{array}{l}\text { Combined } \\
\text { Missing } 2(0)\end{array}$ & $34(3)$ & $9(3)$ & $13(2)$ & $12(4)$ \\
\hline
\end{tabular}

a $120(67 \%)$ were constrained liner or dual mobility.

elective revisions, including revisions due to infection and fracture by indications registered in the DHR. Revision procedures on aseptic indications that turned out to be infected $(\geq 2$ positive intraoperative cultures with the same pathogen from tissue biopsies and subsequent prolonged IV antibiotics treatment) were also excluded. Furthermore, we excluded minor revisions without exchange of femoral or acetabular components (e.g., isolated femoral head and polyethylene liner exchanges). Finally, cases with mismatching operation date $>30$ days between DHR and DNPR were excluded. Hence, 1,345 elective aseptic major component revision hip arthroplasties performed in 1,285 patients were available for analysis (Figure 1).
The revision procedures were divided into total component revisions $(\mathrm{n}=313)$, acetabular component revisions $(n=699)$, and femoral component revisions $(n=333)$.

Indications for revision surgery were aseptic loosening $(63 \%)$, dislocation (14\%), pain (6\%), component failure $(5 \%)$, polyethylene wear $(4 \%)$, osteolysis $(2 \%)$, and other indications (2\%). The indication "other" was scrutinized for potential acute indications (e.g., fracture) and these were excluded.

\section{Statistics}

As all eligible procedures were included; no pre-study power calculation was performed. Continuous variables are given as mean (SD) or median (interquartile range [IQR]) as appropriate. Categorical data is presented as n (\%). Analysis of potential risk factors associated with prolonged LOS $>5$ days and readmission was performed using a multivariable logistic regression model with $95 \%$ confidence intervals (CI). Analysis of missing variables was performed and indicated all missing data was missing at random. Consequently, multiple imputations were used to account for missing values by constructing 5 different imputed datasets and using the averages of these in final analysis (Table 1). The variables included in the risk factor analysis were chosen based on previous studies on preoperative patient characteristics and postoperative morbidity of fast-track primary THA (11). A 2-tailed p-value of < 0.05 was considered to be statistically significant.

Analysis was done using SPSS version 24 (2016; IBM Corp, Armonk, NY, USA).

\section{Ethics, registration, data sharing plan, funding, and potential conflicts of interest}

According to Danish law no approval from the regional ethics committee was needed as the study was noninterventional. The Centre for Fast-Track Hip and Knee Replacement Database is registered as an ongoing study registry on clinicaltrials.gov (NCT01515670). Permission to obtain and store data without informed consent was given from the Danish Patient Safety Authority (3-301356/2/EMJO) and the Danish Data Protection Agency (RH2014-132). Data sharing will be available upon reasonable request to the corresponding author. This research did not receive any specific grant from funding agencies in the public, commercial, or not-for-profit sectors and the authors declare no conflicts of interest in relation to this manuscript.

\section{Results}

Overall, median LOS was 3 days (IQR 2-6) and mean LOS was 5.2 days (SD 7.2) (Table 2), with decreasing LOS from median 6 (IQR 3-10) days in 2010 to 2 (IQR 1-4) days in 2018 (Figure 2). Overall, 30\% of cases had LOS $>5$ days, but 
Table 2. Length of stay (LOS), readmission risk, and mortality. Data given as count (\%) unless otherwise specified

\begin{tabular}{lcccc}
\hline & \multicolumn{5}{c}{$\begin{array}{c}\text { All } \\
\text { revisions } \\
\mathrm{n}=1,345\end{array}$} & $\begin{array}{c}\text { Total } \\
\text { revisions } \\
\mathrm{n}=313\end{array}$ & $\begin{array}{c}\text { componentar } \\
\text { revisions } \\
\mathrm{n}=699\end{array}$ & $\begin{array}{c}\text { Femoral } \\
\text { component } \\
\text { revisions } \\
\mathrm{n}=333\end{array}$ \\
Factor & & & & \\
& $3(2-6)$ & $4(3-7)$ & $3(2-5)$ & $4(2-7)$ \\
Median LOS, days (IQR) & $5(7)$ & $7(8)$ & $4(7)$ & $6(7)$ \\
Mean LOS, days (SD) & $362(27)$ & $117(37)$ & $124(18)$ & $121(36)$ \\
LOS $>5$ days & $176(13)$ & $40(13)$ & $88(13)$ & $48(14)$ \\
Readmission risk $\leq 30$ days & $273(20)$ & $64(20)$ & $138(20)$ & $71(21)$ \\
Readmission risk $\leq 90$ days & $22(2)$ & $6(2)$ & $10(1)$ & $6(2)$ \\
Mortality $\leq 90$ days & & & & \\
\hline
\end{tabular}
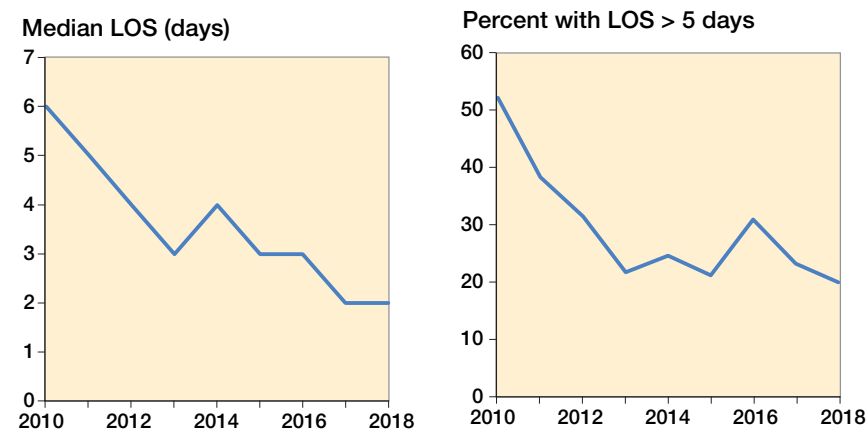

Percent with $\leq 90$ days readmission

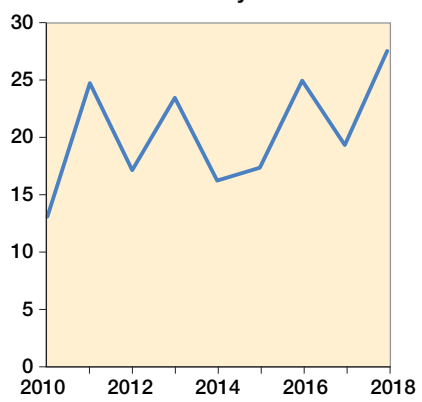

Figure 2. Time-trends and outcomes with changes in median LOS $(A)$, risk of LOS $>5$ days, and risk of readmission $\leq 90$ days during the study period from 2010 to 2018 .

decreasing from $52 \%$ in 2010 to $20 \%$ in 2018 (Figure 2). The most frequent complications associated with $\operatorname{LOS}>5$ days were mobilization problems $(4.4 \%)$ and anemia requiring blood transfusion (4.2\%) (Figure 3). Risk factors for LOS > 5 days were use of walking aid, hemoglobin $\leq 13 \mathrm{~g} / \mathrm{dl}$, cardiac disease, pharmacologically treated psychiatric disorder, age $\geq$ 80 years, age $70-79$ years, $\mathrm{BMI} \geq 35$, and $\geq 1$ previous revision (Table 3, see Supplementary data).

Overall readmission risk was $20 \% \leq 90$ days postoperatively, but fluctuating and increasing from $13 \%$ in 2010 to $28 \%$ in 2018 (Figure 2). The most frequent complications associated with readmission were dislocation $(7.4 \%)$ and periprosthetic joint infection $(2.9 \%)$. Risk factors associated with readmission $\leq 90$ days postoperatively were use of walking aid, hemoglobin $\leq 8 \mathrm{~g} / \mathrm{dL}$, pulmonary disease, pharmacologically treated psychiatric disorder, age $\geq 80$ years (Figure 4, Table 4, see Supplementary data).

$1.6 \%$ patients died $\leq 90$ days postoperatively (Table 5 , see Supplementary data).

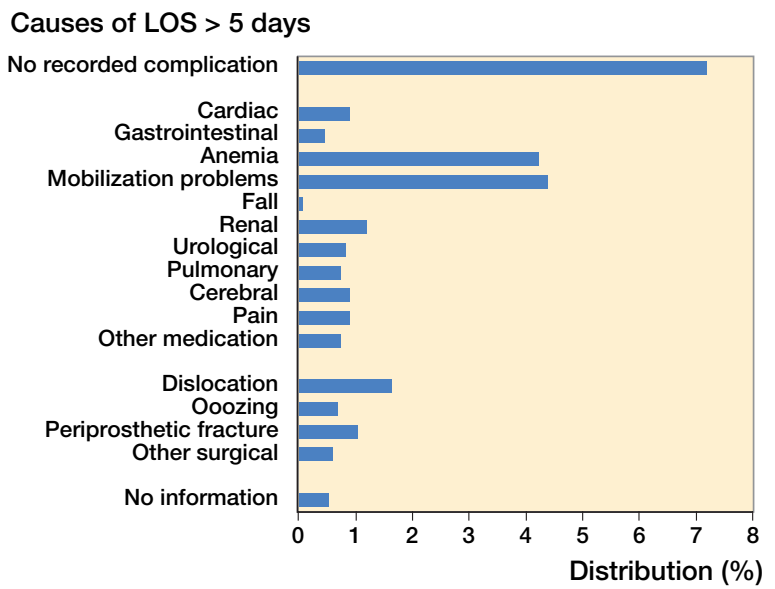

Figure 3. Causes of LOS $>5$ days.

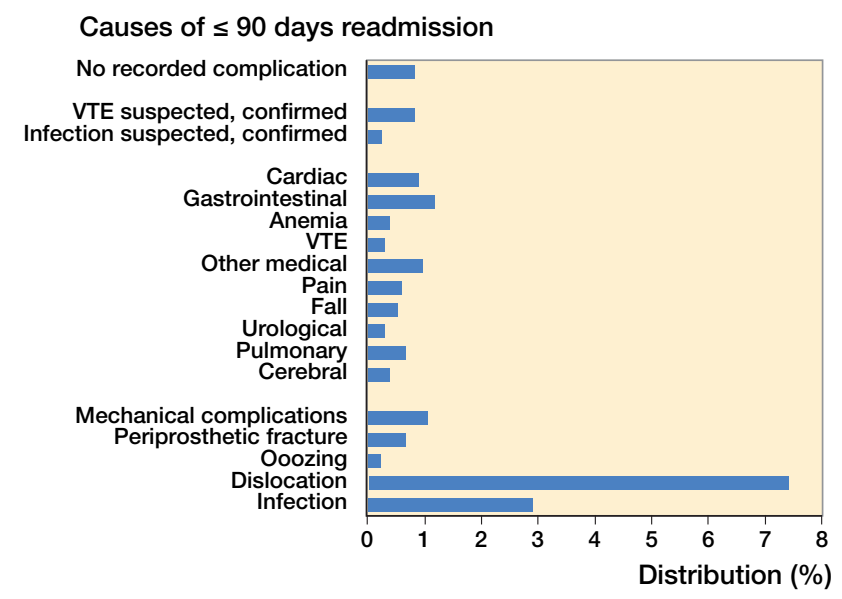

Figure 4. Causes of readmission within 90 days postoperatively.

\section{Discussion}

This is the first multicenter consecutive cohort study on elective aseptic major component R-THA in centers with a wellestablished fast-track protocol in both primary and revision procedures. The main finding of our study was a short and decreasing LOS reaching a median 2 days in 2018, but with a fluctuating high and increasing 90 days readmission rate.

The decreasing LOS was expected and in line with findings on primary fast-track THA procedures from the same period in the same centers and reaching a median 1 day (5). This suggests that fast-track protocols were followed also after R-THA procedures. A median LOS of 3 days in our study is shorter than the 5 days reported in the only previous study on fast-track R-THA procedures $(n=126)(1)$ and shorter than the 5 days in the nationwide Danish data from 2009 to 2011 ( $\mathrm{n}=1,553$ ) (2). However, the overall risk of readmission of $20 \%$ and with increasing tendency during the study period was disappointing. The readmission risk of $20 \%$ is at the same level as reported from the Danish nationwide cohort in 2009-2011 (2). Hence, the recent data indicates 
room for improvement regarding patient safety and postoperative complications.

The most frequent complications associated with increased LOS $>5$ days were anemia and mobilization problems, presumably because of the extensive surgical trauma with high intraoperative blood and bone loss. Postoperative restricted weight-bearing regimes due to complex surgery may also hinder the early mobilization in some cases, and therefore the same fast-track protocol regarding early mobilization as in primary THA may be difficult to follow. Furthermore, the relatively high frequency of patients with postoperative anemia should lead to increased focus on preventive measures and future optimization of perioperative blood management.

The most frequent causes of readmission were surgically related, being early infections (3\%) and dislocations (7\%), which unfortunately is exactly the same frequency as reported in the nationwide study from 2009 to 2011 (2), and demonstrating that the fast-track approach had no beneficial effect on this aspect. A high risk of dislocation (>20\%), especially in cases revised due to previous dislocations, has been described (12). However, if patient outcomes are to improve after R-THA, then a future focus on surgical technique and choice of implants may be needed. Thus, the use of implants decreasing the risk of dislocation (e.g., dual-mobility cup) may be beneficial in more cases and requiring further study (12-14). Furthermore, a direct lateral approach to the hip may be considered in more cases to reduce risk of dislocations (8).

We identified several risk factors for the increased LOS and readmissions including use of walking aid, preoperative hemoglobin $\leq 13 \mathrm{~g} / \mathrm{dL}$, pharmacologically treated psychiatric disorder, age $\geq 80$ years, age 70-79 years (only increased LOS), BMI $\geq 35$ (only increased LOS), and being previously revised (only increased LOS). These are largely similar to primary fast-track procedures (11), which is encouraging as interventions addressing preoperative risk factors prior to primary procedures may also be effective in revision surgery. In particular, the $29 \%$ of patients with hemoglobin $\leq 13 \mathrm{~g} /$ $\mathrm{dL}$ undergoing elective R-THA is a concern. Preoperative anemia is a well-known risk factor for postoperative morbidity after hip and knee arthroplasty surgery and furthermore it is in most cases possible to treat/optimize the condition prior to surgery $(10,15)$. Our findings confirm that patient comorbidity and frailty matters. Such "at-risk patients" may benefit from preoperative identification and use of an extended fast-track/enhanced recovery protocol with increased focus on preoperative optimization and postoperative monitoring rather than on reducing LOS (16). Hence, this should be the main focus in combination with attention to surgical technique/choice of implants in the future optimization of fasttrack protocols in R-THA. Finally, implementing routine use of preoperative high-dose glucocorticoids in aseptic R-THA surgery in all centers could potentially improve outcome (17).
The observational study design is an obvious limitation of this study. Another limitation is unavailable information on cause of readmission in 11 patients and cause of death in 4 patients dying during primary admission. Also, there was a low frequency of missing data on preoperative patient characteristics, but this was adjusted for using multiple imputations.

The major strength of our study is that it is the first study from a large unselected consecutive cohort on fast-track R-THA from a multicenter collaboration with a well-established fast-track set-up in both primary and revision THA in a socialized healthcare setting (5). Also, detailed information on the type of surgical revision strengthens the interpretation of the results. Finally, the prospectively collected data on patient characteristics and the follow-up through a highquality nationwide register using review of discharge notes and patient records for specific causes of morbidity with $>$ 99\% follow-up on readmissions (18) add to the quality of our study.

In conclusion, we found a short and decreasing LOS reaching median 2 days after aseptic major component R-THA, but with a high 90 -day readmission risk $(20 \%)$ with dislocations as the main cause of readmission. Other demonstrated risk factors for postoperative complications suggest that at-risk patients should receive a focus on preoperative optimization and monitoring and less focus on reducing LOS.

MLL, PBP, CJ, and HK wrote the study protocol and analysis plan. MLL, $\mathrm{PBP}$, and $\mathrm{YC}$ undertook data gathering. MLL and CJ performed analysis. MLL wrote the first draft of the manuscript, and all authors and collaborators revised the draft and approved the final manuscript.

The authors would like to thank the research nurses at the participating centers for assistance in gathering patient file information for this study.

Acta thanks Michael Clarius and Stephan Vehmeijer for help with peer review of this study.

1. Joseph Z, Calvert N, Salmon M, Harper M, Swann A, Tan R, et al Enhanced recovery principles applied to revision hip and knee arthroplasty leads to better patient outcomes. J Orthop 2020; 22: 543-7.

2. Lindberg-Larsen M, Jorgensen C C, Hansen T B, Solgaard S, Kehlet H. Early morbidity after aseptic revision hip arthroplasty in Denmark: a two-year nationwide study. Bone Joint J 2014; 96-B(11): 1464-71.

3. Petersen P B, Lindberg-Larsen M, Jorgensen C C, Kehlet H, Lundbeck Foundation Centre for Fast-track Hiip and Knee Arthroplasty collaborating group. Venous thromboembolism after fast-track elective revision hip and knee arthroplasty: a multicentre cohort study of 2814 unselected consecutive procedures. Thromb Res 2021; 199: 101-5.

4. DHR: The Danish Hip Arthroplasty Register, Annual Report 2018. http:// www.dhr.dk/; 2018 (Last accessed August 1, 2021).

5. Petersen PB, Kehlet H, Jorgensen C C, Lundbeck Foundation Centre for Fast-track Hip and Knee Replacement Collaborative Group. Improvement in fast-track hip and knee arthroplasty: a prospective multicentre study of 36,935 procedures from 2010 to 2017. Sci Rep 2020; 10(1): 21233.

6. Husted H. Fast-track hip and knee arthroplasty: clinical and organizational aspects. Acta Orthop Suppl 2012; 83(346): 1-39. 
7. RADS: The Danish Council for the Use of Expensive Hospital Medicines. Treatment recommendation for prevention of venous thromboembolism in hip and knee surgery. http://rads.dk/media/2086/lmrtromboseprofylakse-knae-og-hoftekirurgi-marts-2016.pdf; 2016 (Last accessed August 1,2021).

8. Skoogh O, Tsikandylakis G, Mohaddes M, Nemes S, Odin D, Grant $\mathbf{P}$, et al. Contemporary posterior surgical approach in total hip replacement: still more reoperations due to dislocation compared with direct lateral approach? An observational study of the Swedish Hip Arthroplasty Register including 156,979 hips. Acta Orthop 2019; 90(5): 411-16.

9. Johannesdottir S A, Horvath-Puho E, Ehrenstein V, Schmidt M, Pedersen L, Sorensen H T. Existing data sources for clinical epidemiology: the Danish National Database of Reimbursed Prescriptions. Clin Epidemiol 2012, 4: 303-13.

10. Jans O, Jorgensen C, Kehlet H, Johansson P I, Lundbeck Foundation Centre for Fast-track Hip and Knee Replacement Collaborative Group. Role of preoperative anemia for risk of transfusion and postoperative morbidity in fast-track hip and knee arthroplasty. Transfusion 2014; 54(3): 717-26

11. Jørgensen C C, Petersen M A, Kehlet H, Lundbeck Foundation Centre for Fast-Track Hip and Knee Replacement Collaborative Group. Preoperative prediction of potentially preventable morbidity after fast-track hip and knee arthroplasty: a detailed descriptive cohort study. BMJ Open 2016; 6(1): e009813.
12. Hermansen L L, Viberg B, Overgaard S. Risk factors for dislocation and re-revision after first-time revision total hip arthroplasty due to recurrent dislocation: a study from the Danish Hip Arthroplasty Register. J Arthroplasty 2021; 36(4): 1407-12.

13. Giacomo P, Giulia B, Valerio P, Vincenzo S, Pierluigi A. Dual mobility for total hip arthroplasty revision surgery: a systematic review and metanalysis. SICOT J 2021; 7: 18.

14. Reina N, Pareek A, Krych A J, Pagnano M W, Berry D J, Abdel M P. Dual-mobility constructs in primary and revision total hip arthroplasty: a systematic review of comparative studies. J Arthroplasty 2019; 34(3): 594-603.

15. Saleh E, McClelland D B, Hay A, Semple D, Walsh T S. Prevalence of anaemia before major joint arthroplasty and the potential impact of preoperative investigation and correction on perioperative blood transfusions. Br J Anaesth 2007; 99(6): 801-8.

16. Wainwright $\mathbf{T} \mathbf{W}$, Kehlet H. Fast-track hip and knee arthroplasty: have we reached the goal? Acta Orthop 2019; 90(1): 3-5.

17. Kehlet H, Lindberg-Larsen V. High-dose glucocorticoid before hip and knee arthroplasty: to use or not to use - that's the question. Acta Orthop 2018; 89(5): 477-9.

18. Schmidt M, Schmidt S A, Sandegaard J L, Ehrenstein V, Pedersen L, Sorensen H T. The Danish National Patient Registry: a review of content, data quality, and research potential. Clin Epidemiol 2015; 7 : $449-90$.

\section{Supplementary data}

Table 3. Analysis of risk factors for prolonged LOS > 5 days: multivariate logistic regression analysis

\begin{tabular}{ll}
\hline Risk factors & Odds ratio $(95 \% \mathrm{Cl})$ \\
\hline Acetabular component revision & $0.3(0.3-0.3)$ \\
Femoral component revision & $0.8(0.7-0.9)$ \\
$\quad$ Total revision & 1 (ref.) \\
Previously revised & $1.9(1.6-2.2)$ \\
$\quad$ First-time revisions & 1 (ref.) \\
Use of walking aid & $1.7(1.5-1.9)$ \\
No use of walking aid & 1 (ref.) \\
BMI $\geq 35$ & $2.0(1.6-2.4)$ \\
BMI < 35 & 1 (ref.) \\
Preoperative Hgb $\leq 13$ g/dL & $1.7(1.5-1.9)$ \\
Preoperative Hgb $>13$ g/dL & 1 (ref.) \\
Cardiac disease & $1.4(1.2-1.6)$ \\
No cardiac disease & 1 (ref.) \\
Pulmonary disease & $1.1(0.9-1.3)$ \\
$\quad$ No pulmonary disease & 1 (ref.) \\
Pharmacologically treated & $1.4(1.2-1.6)$ \\
psychiatric disorder & 1 (ref.) \\
No pharmacologically treated & $1.3(0.8-2.0)$ \\
psychiatric disorder & 1 (ref.) \\
Insulin-dependent diabetes & $3.7(3.1-4.4)$ \\
No insulin-dependent diabetes & $2.1(1.8-2.5)$ \\
$\geq 80$ years & 1 (ref.) \\
$70-79$ years & $0.7(0.5-0.9)$ \\
$60-69$ years & $0.8(0.6-1.1)$ \\
$50-59$ years & \\
< 50 years & \\
\hline Cl, confidence interval. &
\end{tabular}

Table 4. Analysis of risk factors for readmission $\leq 90$ days postoperatively: multivariate logistic regression analysis

\begin{tabular}{ll}
\hline Risk factors & Odds ratio $(95 \% \mathrm{Cl})$ \\
\hline Acetabular component revision & $0.9(0.8-1.1)$ \\
Femoral component revision & $0.9(0.8-1.2)$ \\
$\quad$ Total revision & 1 (ref.) \\
Previously revised & $1.0(0.9-1.2)$ \\
First-time revisions & 1 (ref.) \\
Use of walking aid & $1.3(1.1-1.4)$ \\
$\quad$ No use of walking aid & 1 (ref.) \\
BMI $\geq 35$ & $1.1(0.9-1.4)$ \\
BMI <35 & 1 (ref.) \\
Preoperative Hgb $\leq 13$ g/dL & $1.2(1.1-1.4)$ \\
Preoperative Hgb $>13$ g/dL & 1 (ref.) \\
Cardiac disease & $1.0(0.8-1.1)$ \\
No cardiac disease & 1 (ref.) \\
Pulmonary disease & $1.2(1.0-1.4)$ \\
No pulmonary disease & 1 (ref.) \\
Pharmacologically treated & \\
psychiatric disorder & $2.0(1.7-2.3)$ \\
No pharmacologically treated & 1 (ref.) \\
psychiatric disorder & $1.3(0.8-2.3)$ \\
Insulin-dependent diabetes & 1 (ref.) \\
No insulin-dependent diabetes & $1.7(1.4-2.0)$ \\
$\geq 80$ years & $1.1(1.0-1.3)$ \\
$70-79$ years & 1 (ref.) \\
$60-69$ years & $1.0(0.8-1.2)$ \\
$50-59$ years & $0.4(0.3-0.6)$ \\
$<50$ years & \\
\hline Cl, confidence interval. &
\end{tabular}


Table 5. Mortality in 22 patients $(1.6 \%) \leq 90$ days postoperatively, days from surgery, and cause of death

\begin{tabular}{clcl}
\multicolumn{1}{l}{$\begin{array}{l}\text { Days from } \\
\text { surgery }\end{array}$} & Place of death & Age & \multicolumn{1}{c}{ Cause } \\
\hline 1 & Primary admission & 92 & $\begin{array}{l}\text { Cardiac arrest, cause not } \\
\text { specified }\end{array}$ \\
3 & Primary admission & 80 & No information a \\
5 & Primary admission & 76 & Myocardial infarction \\
9 & Home & 75 & Unknown \\
12 & Readmission & 81 & Gastric ulcer \\
14 & Primary admission & 86 & No information a \\
17 & Primary admission & 79 & Cancer-related \\
20 & Readmission & 91 & Pneumonia \\
23 & Primary admission & 82 & No information a \\
23 & Primary admission & 89 & Myocardial infarction \\
26 & Primary admission & 86 & No information \\
32 & Primary admission & 84 & Endocarditis \\
34 & Readmission & 88 & Pneumonia \\
38 & Readmission & 83 & Cancer-related \\
40 & Home & 84 & Unknown \\
42 & Home & 82 & Unknown \\
45 & Home & 86 & Unknown \\
47 & Primary admission & 94 & Fall, femoral fracture \\
47 & Primary admission & 77 & Gastrointestinal bleeding, \\
& & & liver cirrhosis \\
73 & Home & 91 & Unknown \\
78 & Primary admission & 60 & Pneumonia \\
86 & Primary admission & 78 & lleus \\
\hline & &
\end{tabular}

a Missing data in patient files due to change in electronic patient file system. 